\title{
Molecular Characterization of Cryptosporidium spp. Isolated From Immunocompromised Patients and Children
}

\author{
Abdollah Rafiei ${ }^{1,2,}$; Zahra Rashno ${ }^{1}$; Alireza Samarbafzadeh ${ }^{3}$; Shahram Khademvatan ${ }^{1}$ \\ ${ }^{1}$ Department of Medical Parasitology, School of Medicine, Ahvaz Jundishapur University of Medical Sciences, Ahvaz, IR Iran \\ 2 Infectious and Tropical Diseases Research Center, Ahvaz Jundishapur University of Medical Sciences, Ahvaz, IR Iran \\ ${ }^{3}$ Department of Medical Virology, School of Medicine, Ahvaz Jundishapur University of Medical Sciences, Ahvaz, IR Iran \\ ${ }^{*}$ Corresponding author: Abdollah Rafiei, Department of Parasitology, Faculty of Medicine, Jundishapur University of Medical Sciences, Ahvaz, IR Iran. Tel: +98-9161183273, Fax: +98- \\ 6112231325, E-mail: rafieiabdollah@yahoo.com
}

Received: November 19, 2012; Revised: January 29, 2013; Accepted: July 27, 2013

\begin{abstract}
Background: Cryptosporidium is known to be one of the most important causes of diarrhea in children and immunocompromised patients. Genotype characterization of Cryptosporidium species in each region would help in the treatment of this disease, as well as to locate the source of infection and to prevent the disease.

Objectives: This current research was conducted in order to analyze the molecular characterization of isolated Cryptosporidium spp. in the Southwest of Iran.

Materials and Methods: In this survey, 390 fecal samples were collected from immunocompromised individuals and children under five-years-of-age. Parasitic infection was evaluated using wet mount preparation, formalin ether, a modified acid fast staining method and microscopic examination. Finally, a PCR-RFLP assay was performed on the extracted DNA collected from fecal samples that were positive for Cryptosporidium by the acid fast method.

Results: Among the 390 fecal samples, 16 cases (4.1\%) were infected with Cryptosporidium. Molecular and genotype characterization found the following protozoan species; 11 Cryptosporidium parvum (68.8\%), 4 C. hominis (25\%), and one case of C. meleagridis (6.2\%).

Conclusions: The present study emphasized the public health importance of Cryptosporidium spp. in the study area. In addition, it seems that zoonotic species are the most important causes of infection in the region. As far as we are aware this the first report of a C. meleagridis infection in Iran.
\end{abstract}

Keywords:Cryptosporidium; Epidemiology; Genotyping Techniques; RNA, Ribosomal,18S; Iran

\section{Background}

Cryptosporidiosis has been reported in both immunocompetent and immunocompromised patients and it has three major clinical presentations, including; asymptomatic carriage, acute diarrhea, and persistent diarrhea (1). Cryptosporidium spp. infection may cause severe clinical gastrointestinal disorders, especially in people infected with human immunodeficiency virus (HIV)(2), people with malignancies, patients with solid-organ transplants, and hemodialysis patients (3-6). Its prevalence has been reported to be $5-50 \%$ among patients with AIDS (7), and it is also an important cause of AIDS-associated deaths due to severe diarrhea.

According to Plutzer and Karanis (8), 20 Cryptosporidium species have been recognized and approximately 61 Cryptosporidium genotypes have been found with uncertain species status, based on SSU-rRNA sequences. Five Cryptosporidium species/genotypes are responsible for the most common human cryptosporidiosis cases, including; Cryptosporidium hominis, C. parvum, C. meleagridis, $C$. felis, and C. canis (9). Among these species, C. hominis and C. parvum are the most common agents responsible for the majority of human infections, especially in industrialized nations, even though in some areas, the incidence of C. meleagridis infection is as high as C. parvum (10). A few other Cryptosporidium species and genotypes can occasionally cause human infections, including; C. muris, $C$. suis, C. andersoni and C. cervine and genotypes $(9,11,12)$.

The role of each transmission route in endemic areas remains unclear, and this is because of the ability of Cryptosporidium species to infect both humans and a wide variety of animals. In addition to the ubiquitous presence of the organism, the expensive nature of epidemiologic investigations, and the inability to differentiate Cryptosporidium species by conventional microscopic investi-

\section{Implication for health policy/practice/research/medical education:}

The current study demonstrates the high prevalence of predominant zoonotic infections, and revealed that water resources contaminated with animal offal may be the main source of infections in the region. Further studies in different environmental locations are needed in order to better understand the transmission route of Cryptosporidium spp. Our research would enable policy health makers to focus on more accurate risk assessments of environmental and drinking water contamination, and to better educate the public about the risk factors involved in cryptosporidiosis in vulnerable populations.

Copyright (C) 2014,Ahvaz Jundishapur University of Medical Sciences; Published by Kowsar Corp. This is an open-access article distributed under the terms of the Creative Commons Attribution License, which permits unrestricted use, distribution, and reproduction in any medium, provided the original work is properly cited. 
gations, makes this process more difficult (13). Recently the use of molecular methods has significantly helped our understanding of the biology and epidemiology of Cryptosporidium species (14). This includes increased knowledge of the species structure and genetics in the Cryptosporidium population, the roles of various transmission routes in cryptosporidiosis epidemiology, and the significance of parasite genetics in pathogenesis and clinical presentations (15). These recent developments have enabled researchers to provide more accurate risk assessments on environmental and drinking water contamination, and it has also helped health officials to better educate the public about the risk factors involved in the acquisition of cryptosporidiosis in vulnerable populations $(16,17)$. There is little information regarding cryptosporidiosis in the region and no current molecular Cryptosporidium characterization data are available.

\section{Objectives}

The current study was conducted to determine Cryptosporidium genotypes isolated from immunocompromised patients in the Southwest of Iran.

\section{Materials and Methods}

\subsection{Sample Collection}

In this study, 390 stool samples were collected from different groups, including; HIV infected patients, patients with hematological malignancies undergoing chemotherapy, kidney transplant recipients, and children younger than five-years-of-age. A smear was stained using a modified Ziehl-Neelsen method for the detection of Cryptosporidium spp. The positive samples were preserved in $2.5 \%$ potassium dichromate $(\mathrm{K} 2 \mathrm{Cr} 2 \mathrm{O} 7)$ and stored at $4^{\circ} \mathrm{C}$ for DNA extraction.

\subsection{DNA Extraction}

Cryptosporidium oocysts were purified using a sucrose flotation procedure (18). Purified oocysts were washed three times in PBS. Samples were centrifuged at loo0 $\times \mathrm{g}$ for 20 minutes. The pellets were then subjected to $5-8$ freeze-thaw cycles and DNA extraction was carried out using a QIAamp DNA Stool Mini Kit (QIAamp® DNA Stool Mini Kit, USA). Briefly, the total pellet resulting from the sucrose flotation was dissolved in $2 \mathrm{~mL}$ ASL buffer. A total of $1.4 \mathrm{~mL}$ of stool lysate was transferred to a $2 \mathrm{~mL}$ centrifuge tube and incubated at $70^{\circ} \mathrm{C}$ for 15 minutes. All procedures were carried out using a QIAamp DNA Stool Mini Kit according to the manufacturer's instructions.

\subsection{Cryptosporidium Genotyping}

Cryptosporidium oocysts were identified using a smallsubunit rRNA-based on nested PCR, as described by
Xiao et al. (19). Primary PCR was performed by primers SSU-F2: (5 TTCTAGAGCTAATACATGCG 3) and SSU-R2: (5 CCCATTTCCTTCGAAACAGGA 3) (19-22). The primary PCR mixtures contained $10 \mu \mathrm{L}$ of template, 10X PCR buffer, $10 \mathrm{mM}$ deoxynucleoside triphosphate mix (dNTP), $3 \mathrm{mM}$ $\mathrm{MgCL}_{2}, 50 \mathrm{pmol}$ of each primer, and $0.3 \mathrm{U}$ of Taq DNA polymerase in a $50 \mu \mathrm{L}$ reaction volume. The secondary PCR was performed using the following primers SSU-F3: (5 GGAAGGGTTGTATTTATTAGATAAAG 3) and SSU-R4: (5 CTCATAAGGTGCTGAAGGAGTA 3) (21).

The reaction conditions were similar to those described above in the primary PCR, except that $5 \mu \mathrm{L}$ product of the previous PCR was used as the template. Thermocycling parameters were 4 minutes at $94^{\circ} \mathrm{C}$ hot start (initial heat activation step), followed by 35 cycles of 45 seconds at $94^{\circ} \mathrm{C}, 90$ seconds at $58^{\circ} \mathrm{C}$ and 1 minute at $72^{\circ} \mathrm{C}$, with a final extension of 7 minutes at $72^{\circ} \mathrm{C}(19)$. The PCR product was loaded on $1 \%$ agarose gel, electrophoresed for 1 hour. The gel was stained with ethidium bromide and the products were visualized under a UV transilluminator (Uvitec, UK). The size of the amplicon was approximately $830 \mathrm{bp}$.

\subsection{RFLP (Restriction Fragment Lngth Polymor- phism)}

Restriction assays were conducted in a $30 \mu \mathrm{L}$ volume with 0.5 units of restriction enzymes and $20 \mu \mathrm{L}$ of PCR product per reaction. Mixes were incubated at $37^{\circ} \mathrm{C}$ for 8 hours. Digested products were visualized under UV light after $1 \%$ agarose gel electrophoresis and ethidium bromide staining. The endonuclease enzymes used were SspI and VspI (Vivantis, Malaysia).

\section{Results}

Our research showed an incidence of 4.1\% (16/390) Cryptosporidium infection among immunocompromised patients using a microscopic. Higher rates of infection were identified among HIV+ and kidney transplant recipients (Table 1 and Figure 1). RFLP analysis of the PCR products revealed the presence of three Cryptosporidium species in human cases in the Southwest of Iran. The isolates were comprised of; 11 cases of C. parvum, four C. hominis, and one C. meleagridis case (Table 2 and Figure 2 ).

\section{Discussion}

Cryptosporidium can cause a self-limiting infection in immunocompetent cases; on the other hand, it can lead to severe illness in immunocompromised patients. Molecular tools have been developed to detect and differentiate Cryptosporidium species/genotype and its subtype levels $(23,24)$. Our study focused on the current state of Cryptosporidium infection in immunocompromised patients and children younger than five-years-of-age referring to hospitals in Ahvaz City, Southwest of Iran. 
Rafiei A et al.

Table 1. Frequency of Cryptosporidium Species Among Immunocompromised Patients and Children, Southwest of Iran

\begin{tabular}{llll}
\hline Cases & Tested Cases, No. (\%) & Infected Cases, No. $(\%)$ & Cryptosporidium Species \\
\hline Malignant hematologic children & $132(33.8 \%)$ & $4(3)$ & C. hominis, C. parvum \\
Malignant hematologic adults & $111(28.5)$ & $4(3.6)$ & C. hominis, C. parvum \\
Kidney transplant recipients & $48(12.3)$ & $3(6.2)$ & C. hominis, C. parvum \\
HIV+ & $48(12.3)$ & $3(6.2)$ & C.parvum \\
Hemodialysis patients & $32(8.2)$ & $1(3.1)$ & C.parvum \\
Children younger than 5 years & $19(4.9)$ & $1(5.2)$ & C. meleagridis \\
\hline
\end{tabular}

Table 2. Cryptosporidium Species Isolated from Immunocompromised Patients and Children, in the Southwest of Iran

\begin{tabular}{ll}
\hline Cryptosporidium Species & Patients, No.(\%) \\
\hline C.parvum & $11(68.8)$ \\
C.hominis & $4(25)$ \\
C.meleagridis & $1(6.2)$ \\
\hline
\end{tabular}

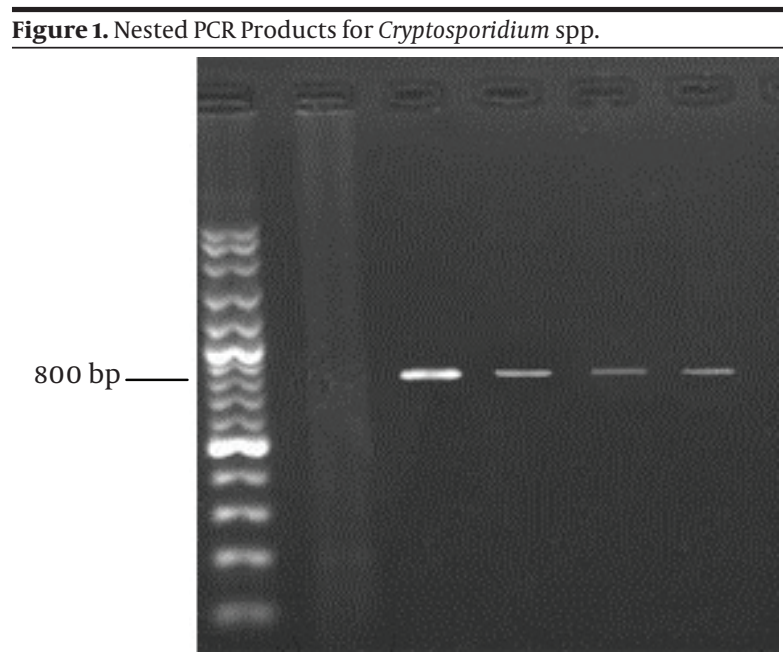

SSUrRNA gene sequences from three patients (lanes 4-6); Lane 1=100 bp marker; Lane 2 = negative control; Lane 3 = positive control for Cryptosporidium.

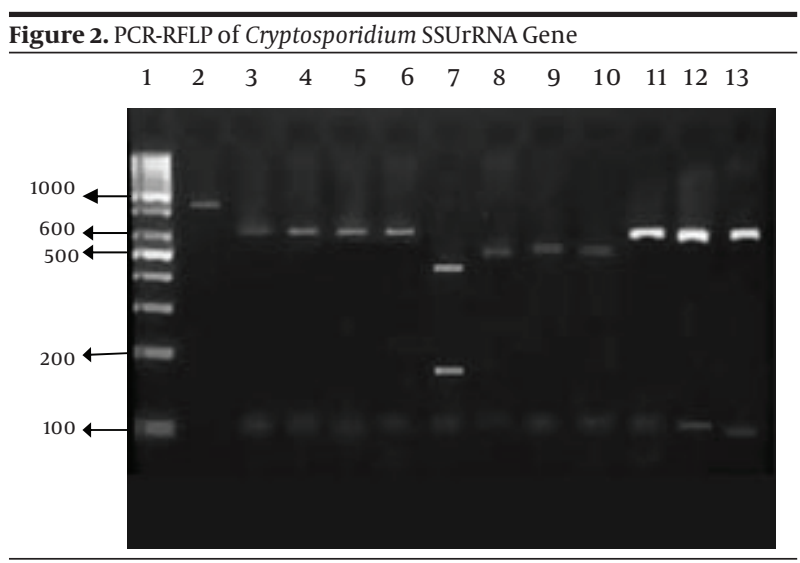

Secondary PCR product was digested by VSPI restriction enzymes. Lane 1, DNA size marker. Lane 2, undigested PCR product. Lanes 3-6 and 11-13, C. parvum 628,104 bp. Lane 7, C. meleagridis 457171 and 115 bp. Lanes 8-10, C. hominis 556 and $116 \mathrm{bp}$.
In the present study, the prevalence of Cryptosporidium was 4.1\% (16/390). A previous study in this region reported a prevalence of 5.1\% (9/179) among immunocompromised patients, which is similar to our report (25). It seems that Cryptosporidium infection is still one of the opportunistic parasitic infections in this region that can result in life threatening disease, especially among immunocompromised patients. After molecular analysis, three different Cryptosporidium species were detected including C. parvum, C. hominis and C. meleagridis. This is the first molecular characterization of Cryptosporidium collected from human patients in the Southwest of Iran. Of the 16 isolates, $68.75 \%$ were C. parvum, $25 \%$ C. hominis and $6.25 \%$ C. meleagridis. The predominance of $C$. parvum may be due to the high prevalence of bovine cryptosporidiosis in this region. The results were in close agreement with previous studies conducted in $\operatorname{Iran}(26,27)$.

Molecular characterization of Cryptosporidium isolates from humans and animals in Iran showed a frequency of $74 \%$ C. parvum and $26 \%$ C. hominis among infected cases in some parts of Iran (26). Additional research in Shahriar (suburb of Tehran) identified 18 out of $24(75 \%)$ isolates from patients with diarrhea as C. parvum (27). These reports were in close agreement with our results. In contrast, in another study C. hominis (71\%) was the most predominant species found among Iranian patients infected with HIV (28). In Saudi Arabia, the incidence of C. parvum was $42.9 \%$, followed by $37 \%$ of C. hominis, C. meleagridis and C. muris in one sample (29). In Kuwait C. parvum and C. hominis were detected in $73 \%$ and $27 \%$ of isolates, respectively (30).

We identified one patient infected with $C$. meleagridis. As far as we are aware this is the first report of a C. meleagridis infection in human hosts in Iran. This species has formerly been reported in other locations, such as; Portugal (31), India (24, 32), Taiwan (33), and the UK (33).

In conclusion, $C$. parvum and C. hominis were the dominant species isolated from immunocompromised patients in the current study. Furthermore, a high incidence of the predominant zoonotic infection in our study revealed that water resource contamination with animal offal may be the main source of infections in the region; therefore, further studies in different environmental areas are needed in order to better understand the route of Cryptosporidium transmission. 


\section{Acknowledgements}

We would like to thank the Health Center staff and all patients who participated in this study.

\section{Authors' Contribution}

Here we confirm that all authors participated in the research design and contributed during different parts of the research.

\section{Financial Disclosure}

All authors declared that they had no conflict of interest.

\section{Funding/Support}

The study was financially supported by the Research Center of Tropical and Infectious Diseases of Ahvaz Jundishapur University of Medical Sciences, Ahvaz, Iran, as a part of the MSc thesis of Miss Zahra Rashno.

\section{References}

1. Xiao L, Ryan UM. Molecular epidemiology. In: Fayer R, Xiao L editors. Cryptosporidium and cryptosporidiosis.. Boca Raton, FL: CRC Press; 2012. p. 119-171.

2. Hunter PR, Hughes S, Woodhouse S, Raj N, Syed Q, Chalmers RM, et al. Health sequelae of human cryptosporidiosis in immunocompetent patients. Clin Infect Dis. 2004;39(4):504-10.

3. Gentile G, Venditti M, Micozzi A, Caprioli A, Donelli G, Tirindelli C, et al. Cryptosporidiosis in patients with hematologic malignancies. Rev Infect Dis. 1991;13(5):842-6.

4. Tanyuksel M, Gun H, Doganci L. Prevalence of Cryptosporidium sp. in patients with neoplasia and diarrhea. Scand J Infect Dis. 1995;27(1):69-70.

5. Sreedharan A, Jayshree RS, Sridhar H. Cryptosporidiosis among cancer patients: an observation. J Diarrhoeal Dis Res. 1996;14(3):211-3.

6. Yang W, Chen P, Villegas EN, Landy RB, Kanetsky C, Cama V, et al. Cryptosporidium source tracking in the Potomac River watershed. Appl Environ Microbiol. 2008;74(21):6495-504.

7. Morgan UM, Deplazes P, Forbes DA, Spano F, Hertzberg H, Sargent $\mathrm{KD}$, et al. Sequence and PCR-RFLP analysis of the internal transcribed spacers of the rDNA repeat unit in isolates of Cryptosporidium from different hosts. Parasitology. 1999;118 ( Pt 1):49-58.

8. Plutzer J, Karanis P. Genetic polymorphism in Cryptosporidium species: an update. Vet Parasitol. 2009;165(3-4):187-99.

9. Xiao L, Zhou L, Santin M, Yang W, Fayer R. Distribution of Cryptosporidium parvum subtypes in calves in eastern United States. Parasitol Res. 2007;100(4):701-6.

10. Cama VA, Ross JM, Crawford S, Kawai V, Chavez-Valdez R, Vargas $\mathrm{D}$, et al. Differences in clinical manifestations among Cryptosporidium species and subtypes in HIV-infected persons.J Infect Dis. 2007;196(5):684-91.

11. Robinson G, Elwin K, Chalmers RM. Unusual Cryptosporidium genotypes in human cases of diarrhea. Emerg Infect Dis. 2008;14(11):1800-2.

12. Feng Y, Ortega Y, He G, Das P, Xu M, Zhang X, et al. Wide geographic distribution of Cryptosporidium bovis and the deer-like genotype in bovines. Vet Parasitol. 2007;144(1-2):1-9.

13. Morgan UM, Xiao L, Fayer R, Lal AA, Thompson RC. Epidemiology and strain variation of Cryptosporidium parvum. Contrib Microbiol. 2000;6:116-39.
14. Xiao L, Fayer R, Ryan U, Upton SJ. Cryptosporidium taxonomy: recent advances and implications for public health. Clin Microbio Rev. 2004;17(1):72-97.

15. Fayer R, Santin M, Trout JM, Greiner E. Prevalence of species and genotypes of Cryptosporidium found in 1-2-year-old dairy cattle in the eastern United States. Vet Parasitol. 2006;135(2):105-12.

16. Xiao L, Fayer R, Ryan U, Upton SJ. Response to the newly proposed species Cryptosporidium pestis. Trends Parasitol. 2007;23(2):41-3.

17. Yosefi F, Rahdar M, Alavi S, Samany A. A study on Prevalence of Gastrointestinal Parasitic Infections in HIV (+) Patients Referred to Ahvaz Razi Hospital in 2008-2009. Jundishapur J Microbiol. 2012:424-6.

18. Coupe S, Sarfati C, Hamane S, Derouin F. Detection of cryptosporidium and identification to the species level by nested PCR and restriction fragment length polymorphism. J Clin Microbiol. 2005;43(3):1017-23.

19. Xiao L, Escalante L, Yang C, Sulaiman I, Escalante AA, Montali RJ et al. Phylogenetic analysis of Cryptosporidium parasites based on the small-subunit rRNA gene locus. Appl Environ Microbiol. 1999;65(4):1578-83.

20. Limor JR, Lal AA, Xiao L. Detection and differentiation of Cryptosporidium parasites that are pathogenic for humans by realtime PCR. JClin Microbiol. 2002;40(7):2335-8.

21. Park JH, Guk SM, Han ET, Shin EH, Kim JL, Chai JY. Genotype analysis of Cryptosporidium spp. prevalent in a rural village in Hwasun-gun, Republic of Korea. Korean J Parasitol. 2006;44(1):27-33.

22. Schindler AR, Abs El-Osta YG, Stevens M, Sinclair MI, Gasser RB Capillary electrophoretic analysis of fragment length polymorphism in ribosomal markers of Cryptosporidium from humans. Mol Cell Probes. 2005;19(6):394-9.

23. Xiao L. Molecular epidemiology of cryptosporidiosis: an update. Exp Parasitol. 2010;124(1):80-9.

24. Xiao L, Bern C, Sulaiman IM, Lal AA. Molecular epidemiology of human cryptosporidiosis. In: Thompson RCA, Armson A, Ryan UM editors. Cryptosporidium: From Molecules to Disease: From Molecules to Disease.. Amsterdam: Elsevier Science; 2004. p.121-146.

25. Dehkordy AB, Rafiei A, Alavi S, Latifi S. Prevalence of cryptosporidium infection in immunocompromised patients, in South-west of iran, 2009-10. Iran J Parasitol. 2010;5(4):42-7.

26. Meamar AR, Guyot K, Certad G, Dei-Cas E, Mohraz M, Mohebali $\mathrm{M}$, et al. Molecular characterization of Cryptosporidium isolates from humans and animals in Iran. Appl Environ Microbiol. 2007;73(3):1033-5.

27. Pirestani M, Sadraei J, Dalimi Asl A, Zavvar M, Vaeznia H. Molecular characterization of Cryptosporidium isolates from human and bovine using 18s rRNA gene in Shahriar county of Tehran, Iran. Parasitol Res. 2008;103(2):467-72.

28. Zavvar M, Sadraei J, Emadi H, Pirestani M. The use of a nested PCRRFLP technique, based on the parasite's $18 \mathrm{~S}$ ribosomal RNA, to characterise Cryptosporidium isolates from HIV/AIDS patients. Ann Trop Med Parasitol. 2008;102(7):597-601.

29. Al-Brikan FA, Salem HS, Beeching N, Hilal N. Multilocus genetic analysis of Cryptosporidium isolates from Saudi Arabia. J Egypt Soc Parasitol. 2008;38(2):645-58.

30. Iqbal J, Khalid N, Hira PR. Cryptosporidiosis in Kuwaiti children: association of clinical characteristics with Cryptosporidium species and subtypes. J Med Microbiol. 2011;60(Pt 5):647-52.

31. Matos O, Alves M, Xiao L, Cama V, Antunes F. Cryptosporidium felis and C. meleagridis in persons with HIV, Portugal. Emerg Infect Dis. 2004;10(12):2256-7.

32. Muthusamy D, Rao SS, Ramani S, Monica B, Banerjee I, Abraham OC, et al. Multilocus genotyping of Cryptosporidium sp. isolates from human immunodeficiency virus-infected individuals in South India. J Clin Microbiol. 2006;44(2):632-4.

33. Pedraza-Diaz S, Amar CF, McLauchlin J, Nichols GL, Cotton KM, Godwin P, et al. Cryptosporidium meleagridis from humans: molecular analysis and description of affected patients. J Infect. 2001;42(4):243-50. 